\title{
Algebraic Topological Analysis of Time-Sequence of Digital Images ${ }^{\star}$
}

\author{
Rocio Gonzalez-Diaz, Belen Medrano*^, Pedro Real, \\ and Javier Sánchez-Peláez \\ Depto. Matematica Aplicada I, E.T.S.I. Informatica, Universidad de Sevilla, \\ Avda. Reina Mercedes, s/n 41012 Sevilla, Spain \\ \{rogodi, belenmg, real\}@us.es \\ http://www.us.es/gtocoma
}

\begin{abstract}
This paper introduces an algebraic framework for a topological analysis of time-varying $2 D$ digital binary-valued images, each of them defined as 2D arrays of pixels. Our answer is based on an algebraictopological coding, called AT-model, for a $n D(n=2,3)$ digital binaryvalued image $I$ consisting simply in taking $I$ together with an algebraic object depending on it. Considering AT-models for all the 2D digital images in a time sequence, it is possible to get an AT-model for the 3D digital image consisting in concatenating the successive $2 \mathrm{D}$ digital images in the sequence. If the frames are represented in a quadtree format, a similar positive result can be derived.
\end{abstract}

\section{Introduction}

In 667, a method for computing cohomology aspects of three--dimensional digital binary-valued images is described. That work is mainly based on two facts: (1)to consider a simplicial model $K(I)$ for a digital image $I$ using a $(14,14)$-adjacency relation between voxels; and (2)to apply an "algebraic homological process" in which an algebraic artifact $c$ (a special type of chain homotopy equivalence [1] ) connecting the chain complex canonically associated to the simplicial version of the digital image with its homology is constructed. An $A T$-model (algebraictopological model) for the $3 D$ digital image $I$ is the couple $(I, c)$. Roughly speaking, an AT-model is an extra algebraic-topological information of the image. This particular description for digital images used there for solving a problem of topological interrogation, is used in this paper for solving a problem of topological analysis. We are interested here in understanding the topological nature of a time-sequence of $2 D$ digital binary-valued images. There are two ways for handling this question: (1) [the intraframe approach] to determine the "topology" of each frame and to try to adequately join these pieces in order to give a correct three-dimensional topological interpretation, or (2) [the $3 D$ approach] to

\footnotetext{
* This work has been partially supported by the PAICYT research project FQM-296 from Junta de Andalucia.

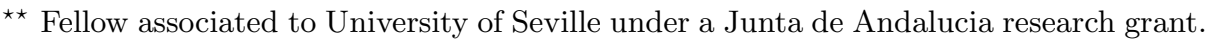


directly obtain the topological information from the $3 D$ image consisting in concatenating the successive $2 D$ images of the sequence. Using AT-models, we will see here that both strategies lead us to the same result. This is also valid in the case in which the $2 D$-images are represented under a quadtree format. To extend these positive results to time-sequences of $3 D$ digital binary-valued images is an extremely interesting challenge which seems to be affordable.

\section{Digital Images and Simplicial Representations}

In this paper, a $n D(n=2,3)$ digital binary-valued image is a $n D$ array of elements (pixels in $2 D$, voxels in $3 D$ ) whose positions are represented by integer coordinates and whose values can only be 1 (black) or 0 (white). Given a pixel $\left(x_{1}, x_{2}\right)$, its 6 -adjacent pixels are $\left(x_{1}-1, x_{2}\right),\left(x_{1}+1, x_{2}\right),\left(x_{1}, x_{2}+1\right),\left(x_{1}+1, x_{2}+\right.$ $1),\left(x_{1}, x_{2}-1\right),\left(x_{1}-1, x_{2}-1\right)$. It is immediate to define another 6 -connectivity if we favour the direction $135^{\circ}$ for determining adjacent pixels instead of $45^{\circ}$. The 6-connectivity in $2 D$ digital images satisfies the Jordan curve property. This ensures that a black simple closed curve (that is, a set of black pixels $C$ such that each pixel in $C$ have exactly two 6-neighbours in $C$ ) will separate the background into two non-6-adjacent white regions, the interior and the exterior. Given a voxel $\left(x_{1}, x_{2}, x_{3}\right)$, its 14-neighbour voxels are showed in Figure 1 . It is possible to define other types of 14-adjacency, favouring other directions. The 14 -adjacency in $3 D$ defined by the neighbour relations given in Figure 1, is an appropriate generalization of the 6 -adjacency in 2D previously defined.

In [7, the 14-adjacency relation is used in order to be able to naturally associate a three-dimensional simplicial complex $K(I)$ to any $3 D$ digital binaryvalued image $I . K(I)$ is called a simplicial representation of $I$ and is defined as a subcomplex of the infinite simplicial complex $K$ obtained by the decomposition of the 3D euclidean space into unit cubes (which vertices are the points $(a, b, c) \in$ $\mathbf{Z}^{3}$ ), and the decomposition of each cube into six tetrahedra as shown in Figure 2 (the six tetrahedra are: $\langle 1,3,4,8\rangle,\langle 1,2,4,8\rangle,\langle 1,2,6,8\rangle,\langle 1,3,7,8\rangle,\langle 1,5,7,8\rangle$, $\langle 1,5,6,8\rangle$.) Two digital images, $I_{1}$ and $I_{2}$, are isomorphic if and only if their simplicial representations $K\left(I_{1}\right)$ and $K\left(I_{2}\right)$ are homeomorphic.

In order to give a formal definition of simplicial representation, we need to give some preliminaries. Our terminology follows [12. Considering an ordered
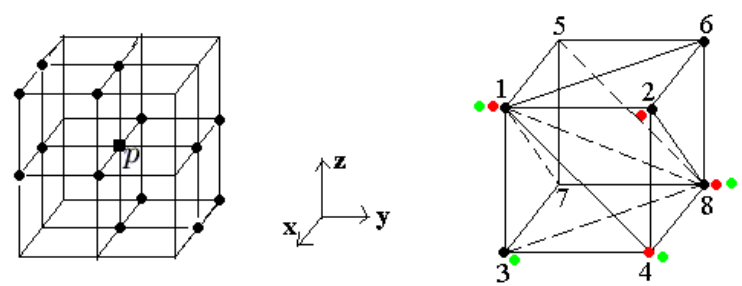

Fig. 1. The 14-neighbours of a voxel $p$ (on the left) and the decomposition of a unit cube into six tetrahedra (on the right) 

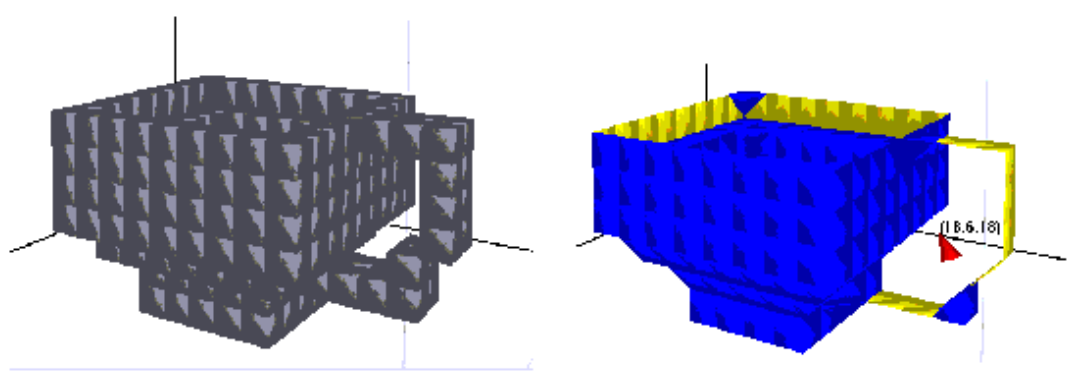

Fig. 2. A digital binary image and its simplicial representation using 14-adjacency relation between voxels

vertex set $V$, a $q$-simplex with $q+1$ affinely independent vertices $v_{0}<\cdots<v_{q}$ of $V$ is the convex hull of these points, denoted by $\left\langle v_{0}, \ldots, v_{q}\right\rangle$. If $i<q$, an $i$-face of $\sigma$ is an $i$-simplex whose vertices are in the set $\left\{v_{0}, \ldots, v_{q}\right\}$. A simplicial complex $K$ is a collection of simplices such that every face of a simplex of $K$ is in $K$ and the intersection of any two simplices of $K$ is a face of each of them or empty. The set of all the $q$-simplices of $K$ is denoted by $K^{(q)}$.

Let $K$ and $L$ be simplicial complexes and let $|K|$ and $|L|$ be the subsets of $\mathbf{R}^{d}$ that are the union of simplices of $K$ and $L$, that is, the geometric realizations of $K$ and $L$, respectively. We say that $K$ and $L$ are homotopic if its respective geometric realization are homotopy equivalents. It is known that if two spaces are homeomorphic then they are homotopy equivalent.

Given a $n D(n=2,3)$ digital binary-valued image $I$ and considering the lexicographical ordering on $\mathbf{Z}^{3}$, a simplicial representation $K(I)$ of $I$ is the simplicial complex described as follows: the $i$-simplices of $K(I)(i \in\{0,1,2,3\})$ are constituted by the different sorted sets of $(i+1)$ 14-neighbour black voxels of $I$. Moreover, let $I$ be a $2 D$ digital binary-valued image embedded in the $3 \mathrm{D}$ digital space. The simplicial representation $K(I)$ of $I$ is the two-dimensional simplicial complex whose $i$-simplices $(i \in\{0,1,2\})$ are constituted by the different sorted sets of $(i+1) 6$-neighbour black pixels of $I$.

\section{An AT-Model for a Digital Image}

In this section we briefly recall the notion of AT-model for digital images given in [677. For explain it, we first define the concept of chain contraction that is an exotic notion in the field of Digital Topology but it is a common resource in Algebraic Topology (see, for example, [11]).

Since the objects considered in this paper are embedded in $\mathbf{R}^{3}$ then the homology groups vanish for dimensions greater than 3 and they are torsionfree for dimensions 0,1 and 2 (see [1-ch.10]). Therefore, for simplicity, we can consider that the ground ring is $\mathbf{Z} / \mathbf{Z} 2$ throughout the paper. Nevertheless, all the procedure we explain here, is valid for any commutative ring. 


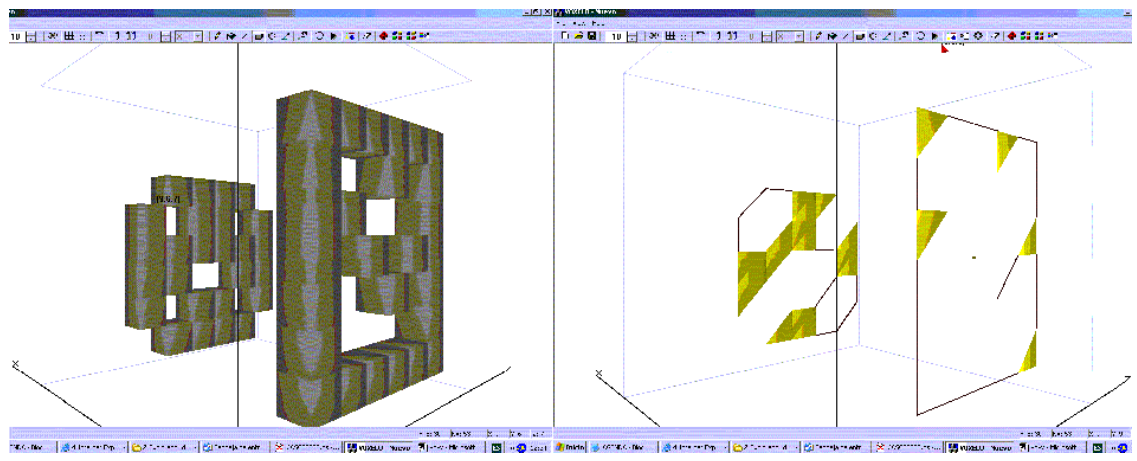

Fig. 3. Two $2 D$ images embedded in the $3 D$ digital space and its respective simplicial representations

Let $K$ be a simplicial complex. A $q$-chain $a$ is a formal sum of simplices of $K^{(q)}$. We denote $\sigma \in a$ if $\sigma \in K^{(q)}$ is a summand of $a$. The $q$-chains form a group with respect to the component-wise addition; this group is the qth chain group of $K$, denoted by $C_{q}(K)$. There is a chain group for every integer $q \geq 0$, but for a complex in $\mathbf{R}^{3}$, only the ones for $0 \leq q \leq 3$ may be non-trivial. The boundary of a $q$-simplex $\sigma=\left\langle v_{0}, \ldots, v_{q}\right\rangle$ is the collection of all its $(q-1)$-faces which is a $(q-1)$-chain: $\partial_{q}(\sigma)=\sum\left\langle v_{0}, \ldots, \hat{v}_{i}, \ldots, v_{q}\right\rangle$, where the hat means that $v_{i}$ is omitted. By linearity, the boundary operator $\partial_{q}$ can be extended to $q$-chains. The collection of boundary operators connect the chain groups $C_{q}(K)$ into the chain complex $C(K)$ canonically associated to $K: \cdots \stackrel{\partial_{2}}{\rightarrow} C_{1}(K) \stackrel{\partial_{1}}{\rightarrow} C_{0}(K) \stackrel{\partial_{0}}{\rightarrow} 0$.

In a more general framework, a chain complex $\mathcal{C}$ is a sequence $\cdots \stackrel{d_{2}}{\longrightarrow} C_{1} \stackrel{d_{1}}{\longrightarrow}$ $C_{0} \stackrel{d_{0}}{\longrightarrow} 0$ of abelian groups $C_{q}$ and homomorphisms $d_{q}$, indexed with the nonnegative integers, such that for all $q, d_{q} d_{q+1}=0$. A chain $a \in C_{q}$ is called a $q$-cycle if $d_{q}(a)=0$. If $a=d_{q+1}\left(a^{\prime}\right)$ for some $a^{\prime} \in C_{q+1}$ then $a$ is called a $q$-boundary. Define the $q$ th homology group to be the quotient group of $q$-cycles and $q$-boundaries, denoted by $H_{q}(\mathcal{C})$. Let $\mathcal{C}=\left\{C_{q}, d_{q}\right\}$ and $\mathcal{C}^{\prime}=\left\{C_{q}^{\prime}, d_{q}^{\prime}\right\}$ be two chain complexes. A chain map $f: \mathcal{C} \rightarrow \mathcal{C}^{\prime}$ is a family of homomorphisms $\left\{f_{q}: C_{q} \rightarrow C_{q}^{\prime}\right\}_{q \geq 0}$ such that $d_{q}^{\prime} f_{q}=f_{q-1} d_{q}$.

Definition 1. 11] $A$ chain contraction of a chain complex $\mathcal{C}$ to another chain complex $\mathcal{C}^{\prime}$ is a set of three homomorphisms $c=(f, g, \phi)$ such that: (i) $f: \mathcal{C} \rightarrow \mathcal{C}^{\prime}$ (called projection) and $g: \mathcal{C}^{\prime} \rightarrow \mathcal{C}$ (called inclusion) are chain maps. (ii) $f g$ is the identity map of $\mathcal{C}^{\prime}$. (iii) $\phi: \mathcal{C} \rightarrow \mathcal{C}$ is a chain homotopy of degree +1 of the identity map $i d_{\mathcal{C}}$ of $\mathcal{C}$ to $g f$, that $i s, i d_{\mathcal{C}}+g f=\phi d+d \phi$, where $d$ is the boundary operator of $\mathcal{C}$.

Important properties of chain contractions are: (a) $\mathcal{C}^{\prime}$ has fewer or the same number of generators than $\mathcal{C}$; (b) $\mathcal{C}$ and $\mathcal{C}^{\prime}$ have isomorphic homology groups [12-p. 73].

Now, we are ready to define an AT-model for a digital image. 
Definition 2. [7] An algebraic topological model (more briefly called AT-model) for a $n D(n=2,3)$ digital image $I$ is the couple $M_{I}=\left(I,\left(f_{I}, g_{I}, \phi_{I}\right)\right)$ where $c_{I}=\left(f_{I}, g_{I}, \phi_{I}\right)$ is a chain contraction of the chain complex $C(K)$ to a chain complex $\mathcal{H}$,being $K$ a simplicial complex homotopic to the simplicial representation $K(I)$ and $\mathcal{H}$ a chain complex isomorphic to the homology of $I$.

It is necessary to emphasize that an AT- model is non-unique. First, $K$ can be any simplicial complex homotopic to the simplicial representation $K(I)$ and $\mathcal{H}$ any chain complex isomorphic to the homology of $I$. Second, the morphisms $f_{I}, g_{I}$ and $\phi_{I}$ can admit different formulas, all of them allowing to define different $c_{I}$ of $C(K)$ to $\mathcal{H}$.

Proposition 1. Let I be a $n D(n=2,3)$ digital binary-valued image. There is an algorithm calculating an AT-model for $I$.

We construct the desired chain contraction adequately modifying the classical algorithms for computing homology (matrix, incremental,...) existing in the literature. For example, the matrix algorithm 12 is based on the reduction of the matrices defining the boundary operator to Smith normal form, from which one can read off the homology groups of the complex. A chain homotopy equivalence version of this process is given in 4. An algebraic homological output for an incremental algorithm [3] for computing homology is given in [6]. The complexity of both algorithms computing AT-models is $O\left(r^{3}\right)$, where $r$ is the number of black pictures elements (pixels or voxels).

Example 1. The algebraic-topological model of the image $J$ showed in Figure 4 is:

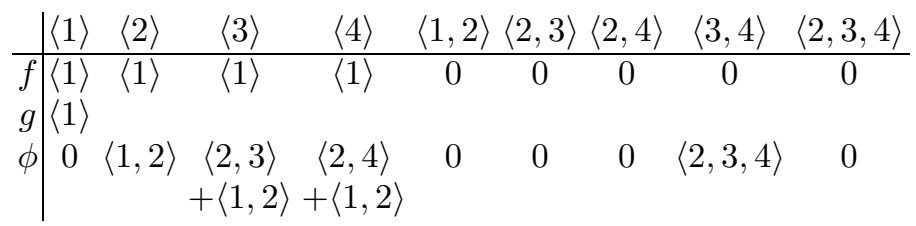

$\mathcal{H}$ has only one generator in dimension 0 represented by the simplex $\langle 1\rangle$. Since $\mathcal{H}$ is isomorphic to $H(J)$ we get that $H(J)$ has only one generator, too.

Let us suppose that we have computed an AT-model $M_{I}=\left(I,\left(f_{I}, g_{I}, \phi_{I}\right)\right.$ for a digital image $I$. We are interested here in a full understanding of the structures and morphisms involved in $M_{I}$. Interesting properties of the morphisms $f_{I}, g_{I}$
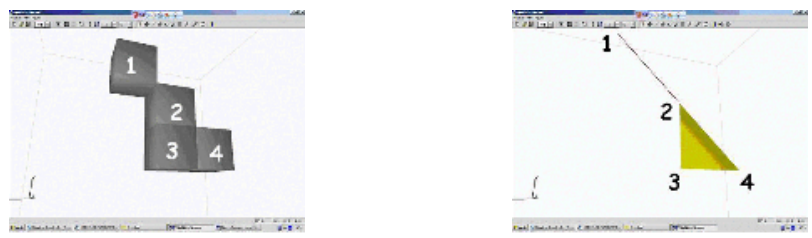

Fig. 4. The black points of the image $J$ and its simplicial representation 
and $\phi_{I}$ are that for all $x \in C(K)$ and $z \in \mathcal{H}$ : (1) $f_{I} \partial(x)=0 ;(2) \partial g_{I}(z)=0$; In fact, $g_{I}(z)$ is a representative cycle of the homology generator $z ;(3) \partial(x)=$ $\partial \phi_{I} \partial(x) ;(4)$ If $\partial(x)=0$, then $x+g_{I} f_{I}(x)=\partial \phi_{I}(x) ;(5)$ If $x=\partial(y)$, then $x=\partial \phi_{I}(x)$.

It is possible to simplify the definition of an AT-model for a $n D$ digital image.

Proposition 2. An AT-model for a $n D(n=2,3)$ digital image $I$ can be represented by a couple $M_{I}=\left(I, \phi_{I}\right)$ where $\phi_{I}: K \rightarrow C(K)$ is a linear map of degree 1 such that $\phi \phi=0, \phi \partial \phi=\phi$ and $\partial \phi \partial=\partial$, where $\partial$ is the boundary operator in $C(K(I)$ and $K$ is a simplicial complex homotopic to the simplicial representation $K(I)$.

Proof. The proof of the previous result is mainly based on two well-known facts. First, given a chain contraction $c=(f, g, \phi)$ from $\mathcal{C}$ to $\mathcal{C}^{\prime}$, it is possible to construct another contraction $c^{\prime}=\left(f, g, \phi^{\prime}\right)$ from $\mathcal{C}$ to $\mathcal{C}^{\prime}$ such that the chain homotopy $\phi^{\prime}$ satisfies the following additional conditions: $(i v) \phi^{\prime} \phi^{\prime}=0 ;(v) \phi^{\prime} g=$ 0 ; $(v i) f \phi^{\prime}=0$. In fact, the formula for $\phi^{\prime}$ is

$$
\phi^{\prime}=(\partial \phi+\phi \partial) \phi(\partial \phi+\phi \partial) \partial(\partial \phi+\phi \partial) \phi(\partial \phi+\phi \partial)
$$

being $\partial$ the boundary operator of $\mathcal{C}$. Second, a chain contraction $c=(f, g, \phi)$ from $\mathcal{C}$ to $\mathcal{C}^{\prime}$ satisfying $(i)-(v i)$ conditions is equivalent to give a map $\phi^{\prime}: \mathcal{C} \rightarrow \mathcal{C}$ (called splitting homotopy) satisfying the following conditions: (1) $\phi^{\prime}$ is a linear map of degree +1 ; (2) $\phi^{\prime} \phi^{\prime}=0$; and $(3) \phi^{\prime} \partial \phi^{\prime}=\phi^{\prime}$, being $\partial$ the boundary operator of $\mathcal{C}$. Let $c=(f, g, \phi)$ be a chain contraction $c=(f, g, \phi)$ from $\mathcal{C}$ to $\mathcal{C}^{\prime}$ satisfying $(i)-(v i)$ conditions. Applying $\phi$ to $(i i i)$ and using the other identities shows that $\phi \partial \phi=\phi$. Then, the desired $\phi^{\prime}$ is $\phi$. Conversely, let $\phi^{\prime}: \mathcal{C} \rightarrow \mathcal{C}$ be a map satisfying $(1)-(3)$. Let $\pi=i d_{\mathcal{C}}-\partial \phi^{\prime}-\phi^{\prime} \partial$. Then, $\mathcal{C}=i m(\pi) \oplus \operatorname{ker}(\pi)$. We define the chain contraction $c^{\prime}=\left(f^{\prime}, g^{\prime}, \phi^{\prime}\right)$ from $\mathcal{C}$ to $i m(\pi)$, where $f^{\prime}$ is the corestriction of $\pi$ and $g^{\prime}$ is the inclusion. Then, using the equality $\pi^{2}=\pi$ and the condition (3), it is easy to prove that $c^{\prime}=\left(f^{\prime}, g^{\prime}, \phi^{\prime}\right)$ is the desired chain contraction.

Now, we are going to prove the proposition. Let $M_{I}=(I,(f, g, \phi))$ be an AT-model for a $n D$ digital image. Then, $c=(f, g, \phi)$ is a chain contraction from $C(K)$ to $\mathcal{H}$, being $\mathcal{H}$ a chain complex isomorphic to the homology of $I$. Using the result previous, we can suppose that the contraction satisfies $(i)-(v i)$ and then we have a splitting homotopy $\phi^{\prime}$ from $C(K)$ to $C(K)$ satisfying (1) - (3). From $\phi^{\prime}$, we have the new chain contraction $c^{\prime}=\left(f^{\prime}, g^{\prime}, \phi^{\prime}\right)$ from $C(K)$ to $i m(\pi)$. Let us now show that $i m(\pi)$ has null boundary operator. If $x=\pi(y) \in i m(\pi)$, then $\partial(x)=\partial \pi(y)=\partial g^{\prime} f^{\prime}(y)=g^{\prime} \partial f^{\prime}(y)=0$ since $\mathcal{H}$ is a chain complex isomorphic to the homology of $I$. Using this last result and applying the operator $\partial$ to condition $\left(\right.$ iii) of $c^{\prime}$, we get the equality $\partial=\partial \partial \phi^{\prime}+\partial \phi^{\prime} \partial$. Since $\partial \partial=0$, we finally obtain that $\partial \phi^{\prime} \partial=\partial$. Conversely, let $\phi: C(K) \rightarrow C(K)$ be a linear map of degree 1 such that $\phi \phi=0, \phi \partial \phi=\phi$ and $\partial \phi \partial=\partial$. We now construct the chain contraction $c=(f, g, \phi): C(K) \rightarrow i m(\pi)$. We are going to show that $c$ defines a AT-model for the digital image $I$. Let $d$ be the boundary operator of 
$i m(\pi)$. We have to prove that $d=0$. Applying $\partial$ to condition (iii) of $c$ and using that $\partial \partial=0$ and $\partial \phi \partial=\partial$, we obtain $\partial-\partial g f=\partial$. Therefore, $\partial g f=g d f=0$. Since $f$ is onto and $g$ is one-to-one, we show that $d=0$.

Briefly, the additional algebraic-topological information showed in a ATmodel for a $n D(n=2,3)$ digital binary-valued image $I$ can be then codified in terms of a chain homotopy satisfying certain conditions. From that algebraic germ, one can form a chain contraction determining the homology of the simplicial complex $K(I)$.

\section{Determining AT-Models Using Other Representation Schemes of Digital Images}

It is possible to compute an AT-model for a digital image using other representation schemes. As an example, let us see how to compute this model using the quadtree representation of a $2 D$ digital binary-valued image.

A quadtree representation (see, for example, [14]) of an image $I$ is a tree whose leaves represent quadrants of the image and are labelled with the color of the corresponding area, i.e, black or white. In order to obtain the quadtree representation of a digital image, first the whole image is decomposed into four equal-sized quadrants. If one of the quadrants does not contain a uniform region (black or white), it is again subdivided into four quadrants. The decomposition stops if only uniform quadrants are encountered. The recursive decomposition is then represented in a data structure known as tree. Each leaf node of a quadtree representation can be assigned a unique locational code corresponding to a sequence of directional codes that locate the leaf node along a path starting at the root of the tree. A black node of a digital image is encoded as $A=\left(A_{1} A_{2} \ldots A_{n}\right)$ with digits in the set $S=\{1,2,3,4\}$ for $A_{i}$, where each digit in the sequence represents the quadrant subdivision from which it originates (see Figure 5 ). The quadrant is defined as the collection of all black node descriptions.

In order to construct the simplicial complex $K(Q(I))$ associated to the quadtree representation $Q(I)$ of the $2 D$ digital image, we need first to find the neighbours of each leaf node of the quadtree $Q(I)$.

Node $B$ is said to be a neighbour of node $A$ in direction $D$ if $B$ corresponds to the block adjacent to $A$ in direction $D$ of size equal, larger or smaller than the block corresponding to $A$. Hence a node can have no neighbour, one or more neighbours in a chosen direction. Using a method similar to that given in 15 and considering 6 -adjacency in a $2 D$ digital image $I$, it is a simple exercise to compute the neighbours of a leaf of the quadtree representation $Q(I)$.

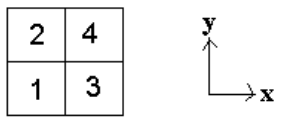

Fig. 5. The quadrant subdivision 
Now, starting from the quadtree representation $Q(I)$ of a digital image $I$, we construct a simplicial complex $K(Q(I))$ as follows: the vertices (or 0-simplices) of $K(Q(I))$ are the leaves of $Q(I)$. The $i$-simplices of $K(Q(I))(i \in\{1,2\})$ are constituted by the different sorted sets of $i+1$ neighbour leaves of $Q(I)$.

Now, it is immediate to see that the geometric realization $|K(Q(I))|$ can be obtained from $|K(I)|$ continuously "deforming" black nodes of $I$ into points.

Proposition 3. The simplicial representation $K(I)$ of a digital image $I$ is homotopic to the simplicial representation $K(Q(I))$ of the quadtree representation $Q(I)$ of $I$.

Since $K(Q(I))$ have, in general, much less number of simplices than $K(I)$, the computation of an algebraic topological model for $I$ using $K(Q(I))$ may be much faster that using $K(I)$.

\section{The Topological Complexity of a Time-Sequence of $2 D$ Digital Images}

We show here that the AT-model technique is well fitted to the problem of analysing the topology of a time-sequence of $2 D$ digital images. Let us recall that there are two ways for handling this question: the intraframe and the $3 D$ approaches.

Let $\left(I_{1}, I_{2}, \ldots, I_{s}\right)$ be a time-sequence of $2 D$ digital binary-valued images. Let $V_{r}$, with $1 \leq r \leq s$, the $3 D$ digital image resulting of concatenating the successive $2 D$ images $I_{1}, I_{2}, \ldots, I_{r}$. This fact is noted by $V_{r}=I_{1}+I_{2}+\ldots+I_{r}$. Let us define an AT-model for a time-sequence of $2 D$ digital images as an ATmodel for the volume $V_{s}$. One method is to directly apply a known algorithm
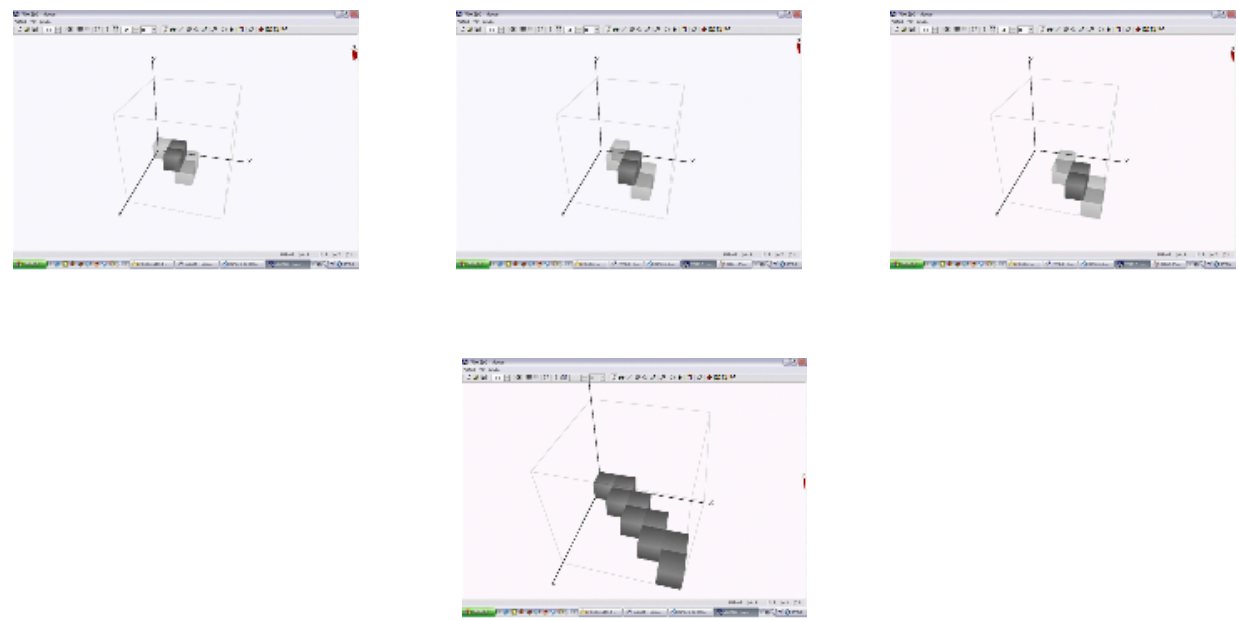

Fig. 6. Several frames of a simple time-sequence and the associated 3D binary image 


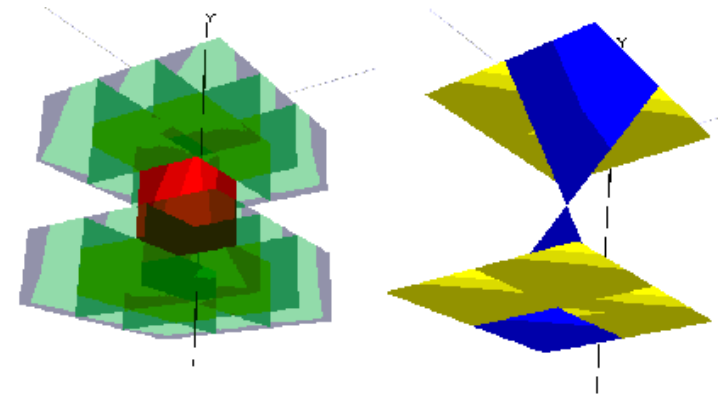

Fig. 7.

for computing an AT-model for the simplicial representation of $V_{s}$. On the other hand, starting from the respective AT-models $\left\{M_{I_{1}}, M_{I_{2}}, \ldots, M_{I_{s}}\right\}$ for all the frames in the sequence, it is possible to adequately "gluing" them in order to form an AT-model for the "concatenated" 3D image $V_{s}$. Let us suppose that we have constructed an AT-model $M_{V_{r}}$ for the $3 D$ image $V_{r}$ (with $r<s$ ). Starting from $M_{V_{r}}$ and $M_{I_{r+1}}$, it is possible to generate an AT-model for $V_{r+1}$. The chain complex $C\left(K\left(V_{r} \cup K\left(I_{r+1}\right)\right)\right)$ coincides with the direct sum of the chain complexes $C\left(K\left(V_{r}\right)\right) \oplus C\left(K\left(I_{r+1}\right)\right)$. It is immediate to construct the chain contraction $c_{V_{r}} \oplus c_{I_{r+1}}$ from $C\left(K\left(V_{r}+I_{r+1}\right)\right)$ to $\left.\left.H\left(K\left(V_{r}\right)\right)\right) \oplus H\left(K\left(I_{r+1}\right)\right)\right)$. Let $f^{r}, g^{r}$ and $\phi^{r}$ be the morphism of that chain contraction and let $h^{r}$ be the set of all the representative homology generators of $H\left(K\left(V_{r}\right) \oplus H\left(K\left(I_{r+1}\right)\right.\right.$. Let $\left\{\tau_{1}, \ldots, \tau_{\ell}\right\}$ be the simplices "connecting" the simplicial complexes $K\left(V_{r}\right)$ and $K\left(I_{r+1}\right)$ such that $\left\{\right.$ simplices of $\left.K\left(V_{r}\right)\right\} \cup\left\{\right.$ simplices of $\left.\left.K\left(I_{r+1}\right)\right)\right\} \cup\left\{\tau_{1}, \ldots, \tau_{r}\right\}$, with $r \leq \ell$ is a subcomplex of $\left.K\left(V_{r+1}\right)\right)$. These simplices are perfectly determined for each voxel in frame $r+1$. As an example, it is showed to the left of Figure 7 , the frame 5 (consisting in one voxel in red) and frames 4 and 6 (consisting respectively in nine voxels in green) of a sequence and to the right we have the simplicial representation of these frames using 14-adjacency relations.

Now, let us use an incremental algorithm for computing an AT-model for a time-sequence of $2 D$ digital binary-valued images knowing AT-models for the frames.

Algorithm 1. InPut: The sorted set of simplices $\left\{\tau_{1}, \ldots, \tau_{\ell}\right\}$ of $K\left(V_{r+1}\right)$ OUTPUT: a splitting homotopy defining an AT-model for the volume $V_{r+1}$ Initially, $f_{\text {alg }}(\sigma):=f^{r}(\sigma), \phi_{\text {alg }}(\sigma):=\phi^{r}(\sigma) \forall \sigma \in K\left(V_{r}\right) \cup K\left(I_{r+1}\right)$; and $h:=h^{r}$ For $i=1$ to $i=\ell$ do

$$
\text { If } \begin{aligned}
& f_{a l g} \partial\left(\sigma_{i}\right)=0 \text { then } \\
& h:=h \cup\left\{\sigma_{i}\right\} \\
& f_{a l g}\left(\sigma_{i}\right):=\sigma_{i} .
\end{aligned}
$$

Else take any one $\sigma_{j}$ of $f_{a l g} \partial\left(\sigma_{i}\right)$, then $h:=h-\left\{\sigma_{j}\right\}$,

For $k=1$ to $k=m$ do

$$
\text { If } \sigma_{j} \text { appears in } f_{a l g}\left(\sigma_{k}\right) \text { then }
$$




$$
\begin{aligned}
& f_{a l g}\left(\sigma_{k}\right):=f_{a l g}\left(\sigma_{k}\right)+f_{a l g} \partial\left(\sigma_{i}\right) \\
& \phi_{\text {alg }}\left(\sigma_{k}\right):=\phi_{\text {alg }}\left(\sigma_{k}\right)+\sigma_{i}+\phi_{\text {alg }} \partial\left(\sigma_{i}\right)
\end{aligned}
$$

\title{
End if.
}

\section{End for.}

\author{
End if. \\ End for. \\ OUTPUT: the splitting homotopy $\phi^{\prime}$ obtained from the homotopy $\phi_{a l g}$.
}

Therefore, we have

Theorem 1. Given a time-sequence $I_{1}, \ldots, I_{s}$ of $2 D$ digital binary-valued images represented as $2 D$ pixel arrays, it is possible to compute an AT-model for the sequence from the information given by the AT-models for all the frames.

If we deal with quadtree representations for the $2 D$ digital images of the sequence, the AT-model technique works well. The key idea is to understand how the simplicial representation $K\left(V_{s}\right)$ based on 14-adjacency relations between voxels of $V_{s}$ is deformed in such a way that each frame $I_{j}(j=1, \ldots, s)$ is represented now by the quadtree simplicial representation $K\left(Q\left(I_{j}\right)\right)$. The resulting simplicial complex of this process is denoted by $K_{Q}\left(V_{s}\right)$. Determining that this simplicial complex is homotopic to the simplicial representation $K\left(V_{s}\right)$ is an elementary question. The intraframe approach here gives the desired AT-model for the volume $V_{s}$. Let us start from AT-models for all quadtree frames in the sequence and take into account that neighbour nodes in frame $j+1$ of a node in frame $j$ are perfectly determined by the 14 -adjacency relations. Then, an similar algorithm to the previous one can be applied to this situation.

Theorem 2. Given a sequence $I_{1}, \ldots, I_{s}$ of $2 D$ digital binary-valued images represented under quadtree format, it is possible to compute an AT-model for the sequence from the information given by the quadtree AT-models for all frames.

\section{Conclusions}

In this paper, we are interested in providing an algebraic solution to the problem of topologically analysing a sequence of 2D digital binary-valued images The method is based on the notion of AT-model for a $n D(n=2,3)$ digital image and both the intraframe and $3 D$ approaches give rise to the same positive results. It seems possible to extend this method to sequences of $3 D$ digital binary-valued images taking into consideration a 30 -adjacency relation between tetraxels (elemental picture elements of dimension 4). In positive case, another interesting question is if the octree representation for $3 D$ digital images could be successfully used in our algebraic-topological setting. On the other hand, an AT-model for a $4 D$ digital image would allow the computation of highly abstract algebraic invariants such as cohomology operations [5] in an efficient way. These new computational tools in the setting of Digital Imaging may be used to facilitate the topological classification problem in $4 D$. 
It is possible to take advantage of temporal coherence of time-varying $2 \mathrm{D}$ digital binary-valued images (coherence between consequent time frames) to obtain a more efficient version of Algorithm 1. Having a time-sequence $\left\{I_{1}, \ldots, I_{s}\right\}$, the idea is to take a "differential" coding of this sequence as, for example, $\left\{I_{1}, D_{2}, D_{3}, \ldots, D_{s}\right\}$, where $D_{i}=I_{i-1} \operatorname{xor} I_{i}$, for all $i=2, \ldots, s$. From an ATmodel for the first frame $I_{1}$, it is possible to gradually generate AT-models for $I_{r}$ and $V_{r}(r=2, \ldots, s)$ by means of a similar method to Algorithm 1. An interesting question would be to determine the complexity of this process.

The results of the previous section show us in particular that an AT-model for a digital image is essentially a reusable datum. In general, from AT-models for simple images, it seems to be possible to directly "manipulate" them in order to obtain an AT-model for a more complex image. To confirm this intuition would be an important result for this theory.

The idea of constructing an "continuous analog" (a polyhedron in this case) reflecting the topology of the digital image as Euclidean subspace goes back to the origin of Digital Topology (see 139] for an introduction to the topics in this area). In order to develop a mathematical theory with high computational and combinatorial flavour, a fundamental goal in this area has been to try to derive purely combinatorial algorithms from the previous algebraic topological scenario. Our method is based on a pure algebraic notion (a chain homotopy equivalence) which is fundamental for both describing the topological complexity of a digital image and enriching the list of digital topological invariants. Further research must be made in order to design an appropriate " digital topology theory" taking as main notion a combinatorial version of a chain homotopy equivalence.

Finally, the application of algebraic topology-based machinery to the field of digital images could be of interest in problems of topological control in Solid Modeling and Computer Aided Geometric Design. An interesting challenge is to know if the AT-model is well adapted to control the topological complexity of a digital image transformed by a digital (local or global) operation.

\section{References}

1. Alexandroff P., Hopf H.: Topologie I. Springer, Berlin 1935

2. Barnes D. and Lambe L.: A fixed approach to homological perturbation theory. Proc. Amer. Math. Soc. 112 (1991) 881-892.

3. Delfinado C.J.A., Edelsbrunner H.: An Incremental Algorithm for Betti Numbers of Simplicial Complexes on the 3-Sphere. Comput. Aided Geom. Design 12 (1995) $771-784$

4. Computation of Cohomology Operations on Finite Simplicial Complexes. Homology, Homotopy and Applications, vol 5(2) (2003) 83-93.

5. González-Díaz R., Real P.: Geometric Object and Cohomology Operations, Proceeding CASC 2002, 121-130.

6. González-Díaz R., Real P.: Towards Digital Cohomology. Lecture Notes in Computer Science vol. 2886 (2003) 92-101.

7. González-Díaz R., Real P.: On the Cohomology of 3D Digital Images. Discrete Applied Math vol. 147, Issues 2-3 (2005) 245-263. 
8. González-Díaz R., Medrano B., Real P., Sánchez-Peláez J.: Topological control in digital images. In preparation.

9. Kong T.Y., Roscoe A.W., Rosenfeld A.: Concepts of Digital Topology. Topology and its Applications 46 (1992) 219-262

10. Lambe L., Stasheff J.: Applications of Perturbation Theory to Iterated Fibrations. Manuscripta Math. 58 (1987) 363-376.

11. MacLane S.: Homology. Classic in Math., Springer-Verlag (1995)

12. Munkres J.R.: Elements of Algebraic Topology. Addison-Wesley Co. 1984

13. Rosenfeld A.: 3D Digital Topology. Inform. and Control 50 (1981) 119-127.

14. Rosenfeld A., Kak A.C.: Digital Picture Processing, vol 2. Academic Press, 1982.

15. Vöros J.: A strategy for repetitive neighbor finding in images represented by quadtrees. Pattern Recognition Letters 18 (1997) 955-962. 